\title{
Structural and vibrational properties of methane up to 71 GPa
}

\author{
Maxim Bykov, ${ }^{1,2}$ Elena Bykova, ${ }^{1}$ Chris J. Pickard, ${ }^{3,4}$ Miguel Martinez-Canales, ${ }^{5}$ Konstantin Glazyrin, ${ }^{6}$ \\ Jesse S. Smith, ${ }^{7}$ and Alexander F. Goncharov $\oplus^{1, *}$ \\ ${ }^{1}$ Earth and Planets Laboratory, Carnegie Institution of Washington, 5251 Broad Branch Road NW, Washington, DC 20015, USA \\ ${ }^{2}$ Howard University, 2400 6th Street NW, Washington, DC 20059, USA \\ ${ }^{3}$ Department of Materials Science \& Metallurgy, University of Cambridge, 27 Charles Babbage Road, Cambridge CB3 OFS, United Kingdom \\ ${ }^{4}$ Advanced Institute for Materials Research, Tohoku University, Aoba, Sendai 980-8577, Japan \\ ${ }^{5}$ School of Physics and Astronomy and CSEC, University of Edinburgh, Peter Guthrie Tait Road, Edinburgh EH9 3FD, United Kingdom \\ ${ }^{6}$ Deutsches Elektronen-Synchrotron DESY, Notkestr. 85, 22607 Hamburg, Germany \\ ${ }^{7}$ HPCAT, X-ray Science Division, Argonne National Laboratory, Argonne, Illinois 60439, USA
}

(Received 29 January 2021; revised 22 September 2021; accepted 25 October 2021; published 8 November 2021)

\begin{abstract}
Single-crystal synchrotron x-ray diffraction, Raman spectroscopy, and first principles calculations have been used to identify the structure of the high-pressure (HP) phase of molecular methane above $20 \mathrm{GPa}$ up to $71 \mathrm{GPa}$ at room temperature. The structure of the HP phase is trigonal $R 3$, which can be represented as a distortion of the cubic phase $B$, previously documented at 7-15 GPa and confirmed here. The positions of hydrogen atoms in the HP phase have been obtained from first principles calculations, which also demonstrated the stability of this structure above $260 \mathrm{~K}$ at $25 \mathrm{GPa}$. The molecules occupy four different crystallographic sites in phase $B$ and 11 sites in the HP phase, which result in splitting of molecular stretching modes detected in Raman spectroscopy and assigned here based on a good agreement with the Raman spectra calculated from the first principles. Our study points out to a single HP phase unlike up to three HP phases proposed previously based on the Raman spectroscopy results only.
\end{abstract}

DOI: 10.1103/PhysRevB.104.184105

\section{INTRODUCTION}

Methane represents a rare and fundamental example of a hydrogen-bearing molecular compound with no hydrogen bonds unlike other simple molecules, $\mathrm{HF}, \mathrm{H}_{2} \mathrm{O}, \mathrm{H}_{2} \mathrm{~S}$, and $\mathrm{NH}_{3}$. Thus, the crystal structure formation in methane solids is expected to be mainly determined by the steric effects and van der Waals bonds, suggesting that its behavior at high pressure may be similar to the rare gas solids, for example Ar [1], which persists in a face-centered-cubic (fcc) structure to very high pressures. Furthermore, the high-pressure behavior of methane is of interest to explore possible deviations from simple closed packed structures and related to this molecular orientational ordering, where the hydrogen atoms find the most energetically favorable stable positions. The fate of methane at even higher compression states in the regime of molecular proximity (i.e., shortened intermolecular $\mathrm{H}-\mathrm{H}$ distances) is of great interest as it can be considered as a hydrogen dominant alloy [2] with the relevance to hightemperature superconductivity [3]. In addition, the behavior of methane at high pressures and temperatures may be of interest because it is believed to be a valued component of the deep planetary interiors of "icy" giant planets such as Uranus and Neptune [4]. Such hot molecular ices of water and ammonia form superionic crystals with diffusive protons at extreme pressure-temperature $(P-T)$ conditions of relevance for these

\footnotetext{
*agoncharov@carnegiescience.edu
}

planets [5-7], while methane tends to chemically react yielding oligomeric hydrocarbon compounds [8] and dissociate at higher $P-T$ conditions yielding diamond and hydrogen [9-11].

Similar to Ar, methane crystallizes in phase I at about 1.5 GPa upon compression at room temperate forming a facecentered-cubic (fcc) closed packed structure of the molecular centers at the carbon atoms [12], and this crystal is plasticmolecules are freely rotating. However, unlike in noble gases, this structure transforms at $5.4 \mathrm{GPa}$ to a complex rhombohedral structure $A$ with 21 molecules in the unit cell solved by a combination of single crystal neutron and $\mathrm{x}$-ray diffraction (XRD) techniques [13]. The structure is rhombohedrally distorted from the fcc methane I, with the carbon atoms occupying nine different crystallographic positions, and some of them may be associated with orientationally ordered molecules. However, the Raman and IR spectra do not show the obvious splitting of the major $v_{1}$ and $v_{3} \mathrm{C}-\mathrm{H}$ stretching modes [14].

Further compression leads to a transition to phase $B$ with a very large unit cell containing 58 molecules, which has been found cubic as solved recently by Maynard-Casely et al. [15] for the carbon subsystem by combining powder and singlecrystal XRD. The phase $B$ shows a splitting of $v_{1}$ and $v_{3}$ stretching C-H modes in IR and Raman spectra [1,14,16,17], suggesting that it possesses some elements of orientational order (e.g., Refs. $[14,16]$ ). The transition from phase $A$ to phase $B$ at about $8 \mathrm{GPa}$ is very sluggish and phase $B$ can be missed if fast compressed yielding a simple cubic phase pre- $B$ [16], where the Raman active $v_{1}$ and $v_{3} \mathrm{C}-\mathrm{H}$ stretching 


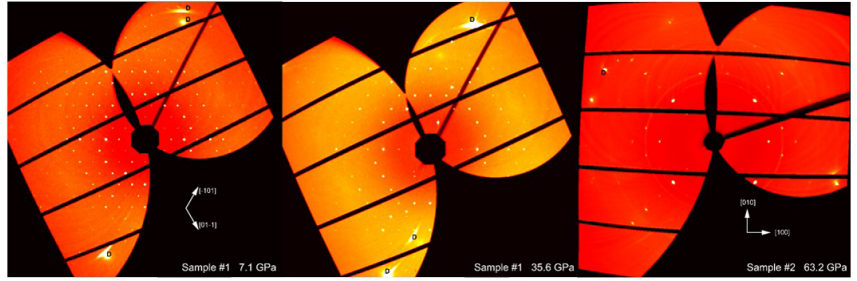

FIG. 1. Reconstructed reciprocal lattice plane of methane visualized by CRYSALISPRO software package [24] at 7.07 GPa (left), 35.6 $\mathrm{GPa}$ (middle), and 63.2 GPa (right). D stands for diamond reflections. The observed diffraction spots from the sample have been indexed and used to determine the structure of phases $B$ (left panel) and high-pressure (HP) phase (middle and right panels) (Table S1 and Tables I and II, respectively).

modes are not split, suggesting that methane molecules are orientationally disordered. Phase $B$ is reported to transform to a high-pressure phase 1 (HP1) at about $25 \mathrm{GPa}$ manifested by anomalies in IR spectra, vibrational frequencies [18], and specific volume [19], while no drastic structural changes were detected in the powder diffraction patterns. At higher pressures, Raman spectroscopy observations suggest further structural modifications to HP2 and HP3 phases reported up to $62 \mathrm{GPa}[16]$; however, the transition pressures to the subsequent high-pressure phases are contradictory $[16,17]$. The structure of the HP phase(s) has not been solved; it has been postulated to be cubic (e.g., Ref. [20]), but no detailed structural information is available. The XRD experiments have been extended to $202 \mathrm{GPa}$ and the volume has been determined based on a cubic symmetry, even though a major transformation has been detected and a volume discontinuity was found at $94 \mathrm{GPa}$ [20]. Methane was reported to persist in a molecular crystal structure beyond $200 \mathrm{GPa}[20,21]$.

In this paper, we examine the structure of methane in the pressure range 7-71 GPa using single-crystal XRD, Raman spectroscopy, and first principles theoretical calculations. Our single-crystal XRD data directly determine the structure of the carbon subsystem in agreement with the previous report in phase $B$ [15], while we find that the HP phase can be viewed as a rhombohedrally distorted phase $B$ (space group $R 3$ ) with 87 molecules in the unit cell (29 in the primitive unit cell); this structure remains unchanged between 20 and $73 \mathrm{GPa}$. We applied an ab initio random structure search of the most stable structures with and without any restriction of the symmetry. The results yield the structure of HP phase in agreement with XRD data, and determine the hydrogen positions in HP phase. The Raman spectrum of this phase calculated from the first principles is in a qualitative agreement with the experiment demonstrating that the $\mathrm{C}-\mathrm{H}$ stretching mode splitting is due to a variation in the crystal field at different crystallographic sites.

\section{EXPERIMENTAL AND THEORETICAL PROCEDURES}

Methane gas was loaded in a mixture with He gas at approximately $1: 1$ mole ratio compressed to $0.15 \mathrm{GPa}$ at room temperature in a BX90 diamond anvil cell [22] (Fig. S1 of the Supplemental Material [23]). In order to obtain good-quality single crystals of phase $B$ of methane, the gas mixture was
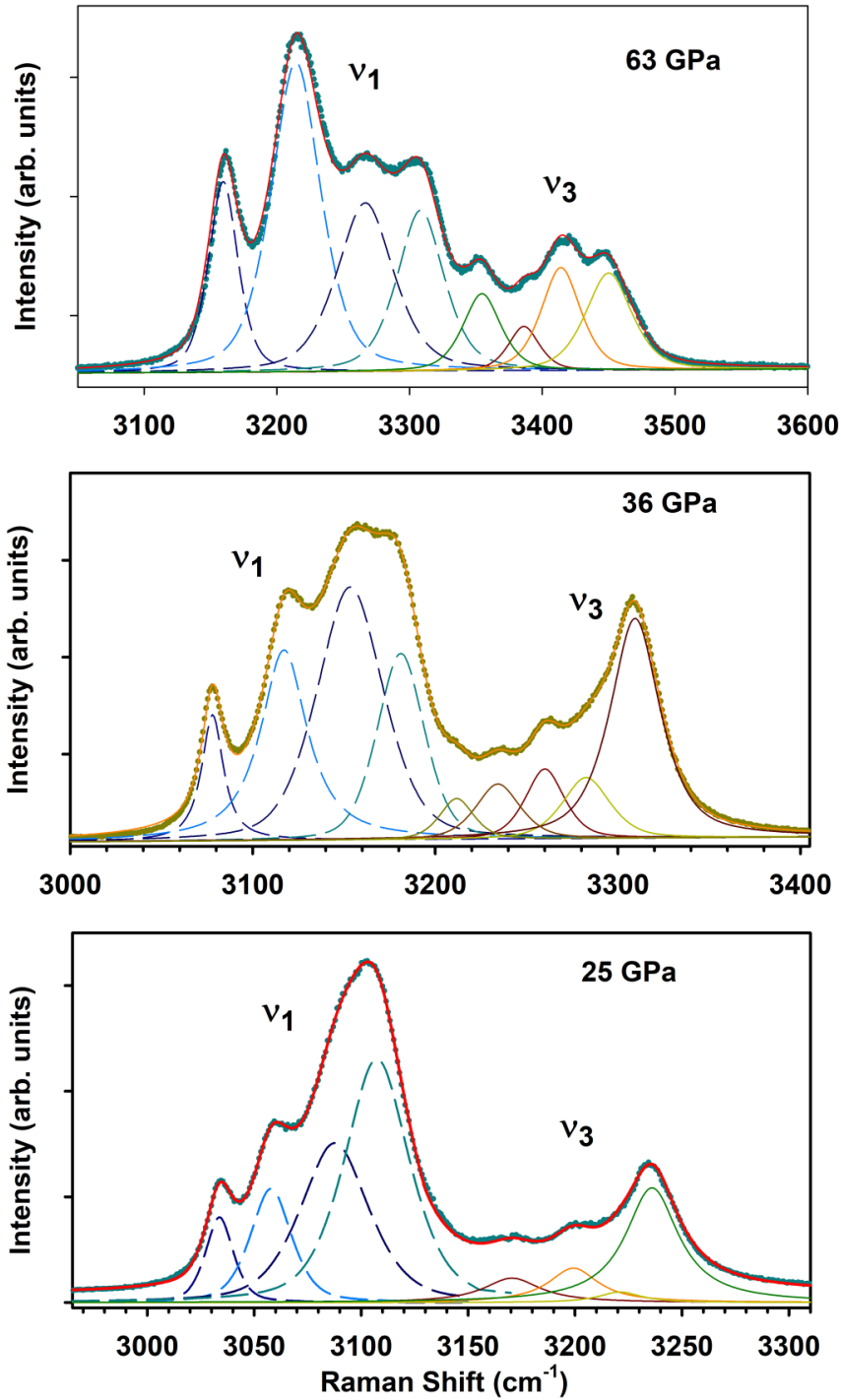

FIG. 2. Raman spectra of the C-H stretching modes of methane in HP phase at various pressures. The dots are the data and the lines are the fits to the Voigt oscillator model with eight (nine for the 36-GPa spectrum) peaks; variable color lines show the individual $v_{1}$ (dashed) and $v_{3}$ (solid) components. Note a change in the frequency scale in different panels due to a pressure shift.

slowly compressed at $297 \mathrm{~K}$ through the methane solidification point at $1.5 \mathrm{GPa}$ and then to $8 \mathrm{GPa}$. At this pressure, excellent single crystals of phase $B$ were grown overnight judging on Raman observations of a splitting of the $v_{1} \mathrm{C}-\mathrm{H}$

TABLE I. Comparison of agreement factors between $R 3$ and $R 3 m$ models of the HP methane.

\begin{tabular}{lcccccc}
\hline \hline & & \multicolumn{2}{c}{$R 3$ model } & & \multicolumn{2}{c}{$R 3 m$ model } \\
\cline { 6 - 7 } $\begin{array}{l}\text { Pressure } \\
\text { GPa }\end{array}$ & $\begin{array}{c}\text { No. of } \\
\text { reflections }\end{array}$ & $\begin{array}{c}\text { No. of } \\
\text { parameters }\end{array}$ & $R_{1}$ & & $\begin{array}{c}\text { No. of } \\
\text { parameters }\end{array}$ & $R_{1}$ \\
\hline 22.4 & 381 & 41 & 0.127 & & 28 & 0.1303 \\
27.4 & 474 & 41 & 0.138 & & 28 & 0.1422 \\
35.6 & 280 & 41 & 0.1020 & & 28 & 0.1033 \\
39.0 & 198 & 41 & 0.1415 & & 28 & 0.1436 \\
\hline \hline
\end{tabular}


TABLE II. Crystal data, refinement and crystal structure details of Methane HP phase.

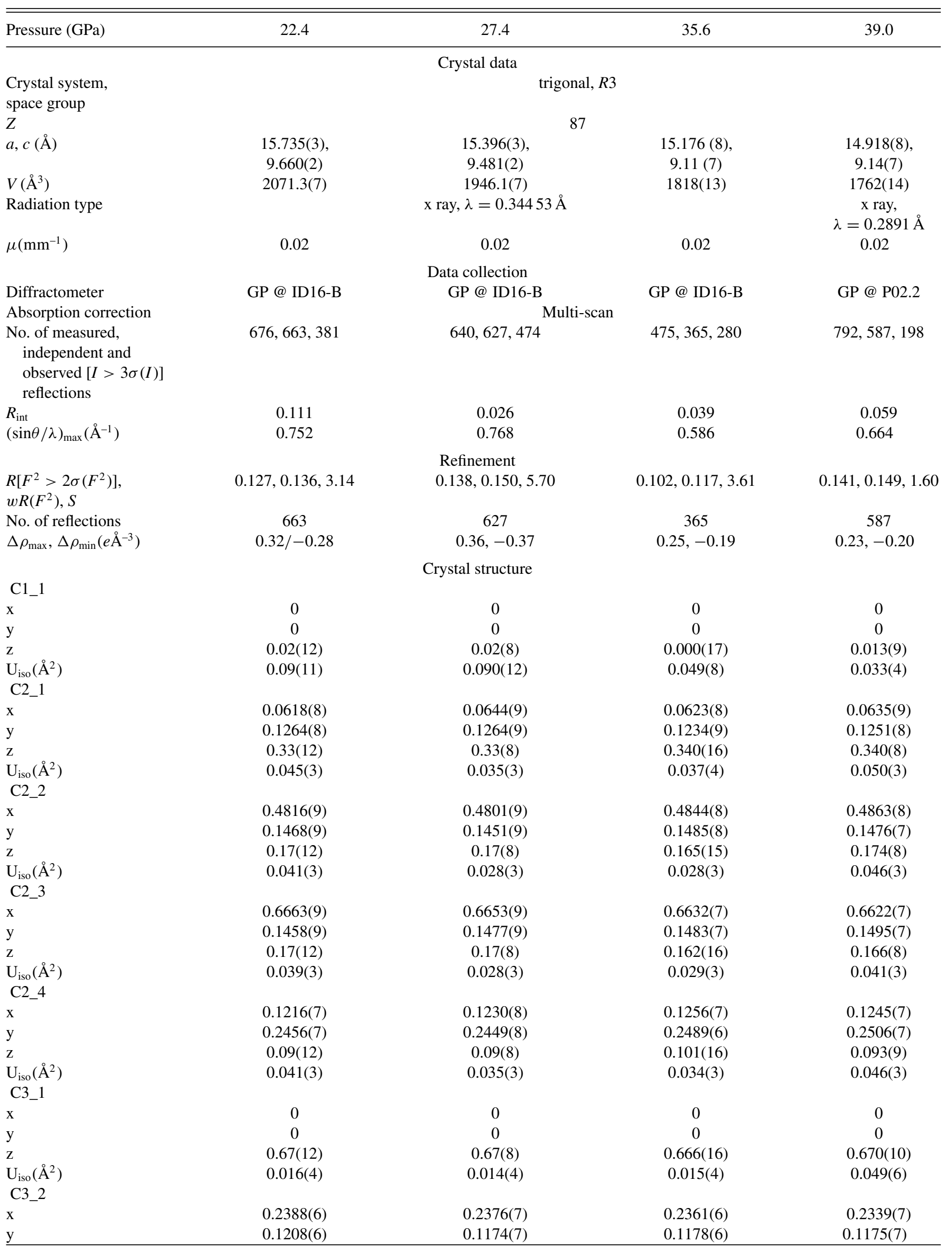


TABLE II. (Continued.)

\begin{tabular}{|c|c|c|c|c|}
\hline $\mathrm{z}$ & $0.14(12)$ & $0.14(8)$ & $0.152(15)$ & $0.156(8)$ \\
\hline $\mathrm{U}_{\text {iso }}\left(\AA^{2}\right)$ & $0.018(2)$ & $0.014(2)$ & $0.019(3)$ & $0.039(3)$ \\
\hline \multicolumn{5}{|l|}{ C4_1 } \\
\hline $\mathrm{x}$ & $0.4644(8)$ & $0.4595(8)$ & $0.4648(7)$ & $0.4635(7)$ \\
\hline y & $-0.0756(7)$ & $-0.0774(8)$ & $-0.0704(6)$ & $-0.0730(7)$ \\
\hline \multicolumn{5}{|l|}{ C4_2 } \\
\hline $\mathrm{x}$ & $0.0092(9)$ & $0.0123(10)$ & $0.0065(9)$ & $0.0062(7)$ \\
\hline $\mathrm{y}$ & $0.3087(10)$ & $0.3130(11)$ & $0.3133(9)$ & $0.3104(7)$ \\
\hline $\mathrm{z}$ & $0.33(12)$ & $0.33(8)$ & $0.339(16)$ & $0.337(8)$ \\
\hline $\mathrm{U}_{\text {iso }}\left(\AA^{2}\right)$ & $0.050(4)$ & $0.049(4)$ & $0.036(3)$ & $0.038(3)$ \\
\hline \multicolumn{5}{|l|}{ C4_3 } \\
\hline \multicolumn{5}{|l|}{ C4_4 } \\
\hline $\mathrm{x}$ & $0.2294(8)$ & $0.2239(10)$ & $0.2251(8)$ & $0.2230(8)$ \\
\hline y & $0.4529(8)$ & $0.4531(10)$ & $0.4496(9)$ & $0.4481(8)$ \\
\hline $\mathrm{z}$ & $0.19(12)$ & $0.18(8)$ & $0.166(15)$ & $0.154(8)$ \\
\hline $\mathrm{U}_{\mathrm{iso}}\left(\AA^{2}\right)$ & $0.038(3)$ & $0.039(3)$ & $0.030(3)$ & $0.040(3)$ \\
\hline
\end{tabular}

stretch mode (Fig. S2 of the Supplemental Material [23]). These crystals yield very sharp Bragg reflections with typical rocking-curve width of $0.64^{\circ}$ at $7.1 \mathrm{GPa}$ (Fig. 1). Two samples (nos. 1 and 2) were compressed up to 39 and $71 \mathrm{GPa}$ respectively and concomitant XRD and Raman spectroscopy measurements (Figs. 1 and 2 and Fig. S2 of the Supplemental Material [23]) were taken at selected pressure points. All the experiments were performed at room temperature [295(2) K].

X-ray diffraction was performed at the beamlines 16-ID-B (APS, Argonne, USA) and P02.2 (Petra III, DESY, Hamburg, Germany). The following beamline setups were used. P02.2: $\lambda=0.289 \AA$, beam size $\sim 2 \times 2 \mu \mathrm{m}^{2}$, Perkin Elmer
XRD 1621 detector; 16-ID-B: $\lambda=0.344 \AA$, beam size $\sim 3 \times 3 \mu \mathrm{m}^{2}$, Pilatus $1 \mathrm{M}$ detector. For the single-crystal XRD measurements samples were rotated around a vertical $\omega$ axis in a range $\pm 22^{\circ}$. The diffraction images were collected with an angular step $\Delta \omega=0.5^{\circ}$ and an exposure time of 2-10 s/frame. For analysis of the single-crystal diffraction data (indexing, data integration, frame scaling and absorption correction) we used the crysalis ${ }^{\text {pro }}$ software package [24]. To calibrate an instrumental model in the crysalis ${ }^{\text {pro }}$ software, i.e., the sample-to-detector distance, detector's origin, offsets of goniometer angles, and rotation of both x-ray beam and the detector around the instrument axis, we used a
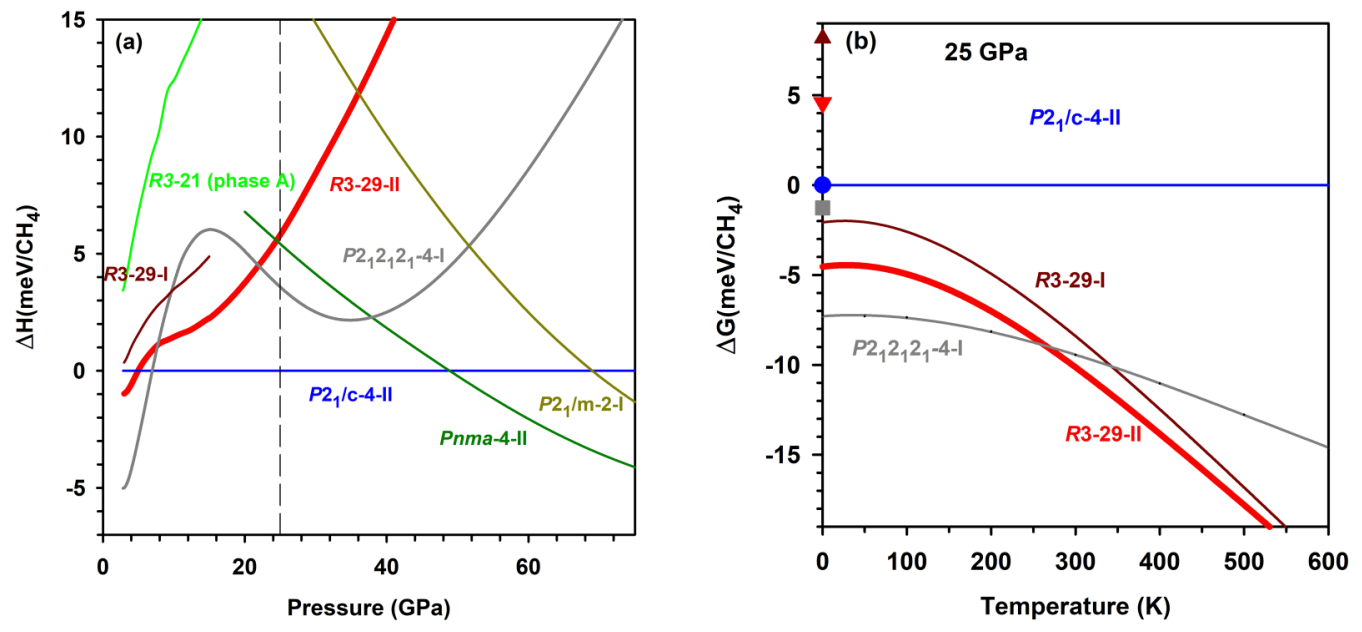

FIG. 3. (a) Theoretically calculated relative enthalpy of the most stable methane phases. The results for HP phase (R3-II) are shown by a thick red line. Other orientationally ordered phases are shown by thin lines of various colors. The vertical dashed line correspond to the pressure at which the temperature effects were taken into account. (b) Relative Gibbs free energies of the most stable methane phases as a function of temperature at $25 \mathrm{GPa}$. The symbols at $T=0 \mathrm{~K}$ represent relative classical enthalpies as computed with norm-conserving pseudopotentials. 
TABLE III. Crystal structure of $\mathrm{HP}^{-\mathrm{CH}_{4}}$ at $25 \mathrm{GPa}$ optimized by calculations $(R 3, a=15.3315, c=9.5221 \AA)$.

\begin{tabular}{|c|c|c|c|}
\hline Site & $x$ & $y$ & $z$ \\
\hline C1_1 & 0 & 0 & -0.9996 \\
\hline H1_1_1 & -0.365771 & -0.622456 & -0.628642 \\
\hline H1_1_2 & 0 & 0 & -0.11349 \\
\hline C2_1 & -0.131193 & -0.066507 & -0.68994 \\
\hline $\mathrm{H} 2 \_1 \_1$ & -0.181504 & -0.146561 & -0.695142 \\
\hline $\mathrm{H} 2 \_1 \_2$ & -0.083784 & -0.042373 & -0.781794 \\
\hline $\mathrm{H} 2 \_1 \_3$ & -0.085473 & -0.048146 & -0.596947 \\
\hline H2_1_4 & -0.174924 & -0.029387 & -0.685741 \\
\hline $\mathrm{C} 2 \_2$ & 0.148245 & 0.474857 & -0.519002 \\
\hline $\mathrm{H} 2 \_2 \_1$ & 0.079276 & 0.476659 & -0.509461 \\
\hline $\mathrm{H} 2 \_2 \_2$ & 0.205456 & 0.534268 & -0.456087 \\
\hline $\mathrm{H} 2 \_2 \_3$ & 0.136305 & 0.402921 & -0.484066 \\
\hline $\mathrm{H} 2 \_2 \_4$ & -0.162001 & -0.181677 & -0.294102 \\
\hline $\mathrm{C} 2 \_3$ & -0.183006 & 0.005534 & -0.191981 \\
\hline H2_3_1 & -0.255161 & -0.062329 & -0.199231 \\
\hline $\mathrm{H} 2 \_3 \_2$ & 0.144078 & -0.273369 & -0.461258 \\
\hline $\mathrm{H} 2 \_3 \_3$ & -0.129179 & -0.010913 & -0.144596 \\
\hline $\mathrm{H} 2 \_3 \_4$ & -0.157189 & 0.036738 & -0.29511 \\
\hline $\mathrm{C} 2 \_4$ & -0.252732 & -0.13696 & -0.448695 \\
\hline $\mathrm{H} 2 \_4 \_1$ & -0.285273 & -0.116555 & -0.534236 \\
\hline $\mathrm{H} 2 \_4 \_2$ & -0.300828 & -0.15757 & -0.357472 \\
\hline $\mathrm{H} 2 \_4 \_3$ & -0.243439 & -0.199203 & -0.480598 \\
\hline $\mathrm{H} 2 \_4 \_4$ & -0.180176 & -0.073615 & -0.422955 \\
\hline C3_1 & 0 & 0 & -0.346612 \\
\hline H3_1_1 & 0.044147 & -0.032218 & -0.309195 \\
\hline $\mathrm{H} 3$ _1_2 & 0 & 0 & -0.460331 \\
\hline C3_2 & -0.096727 & -0.545671 & -0.549019 \\
\hline H3_2_1 & 0.173063 & 0.094993 & -0.812157 \\
\hline H3_2_2 & -0.079162 & -0.473773 & -0.593158 \\
\hline H3_2_3 & -0.447561 & -0.266257 & -0.299235 \\
\hline H3_2_4 & 0.299905 & 0.127484 & -0.823554 \\
\hline C4_1 & -0.207481 & -0.422449 & -0.598684 \\
\hline H4_1_1 & -0.274275 & -0.422628 & -0.563519 \\
\hline H4_1_2 & -0.226509 & -0.496916 & -0.631667 \\
\hline H4_1_3 & -0.173923 & -0.37107 & -0.685189 \\
\hline H4_1_4 & -0.154241 & -0.399094 & -0.513821 \\
\hline C4_2 & -0.322138 & -0.356324 & -0.361957 \\
\hline H4_2_1 & -0.047361 & 0.238783 & -0.656792 \\
\hline H4_2_2 & 0.083251 & 0.322741 & -0.659402 \\
\hline H4_2_3 & -0.33452 & -0.297433 & -0.322463 \\
\hline H4_2_4 & -0.323976 & -0.355022 & -0.47503 \\
\hline C4_3 & -0.316752 & -0.01339 & -0.682065 \\
\hline H4_3_1 & -0.308246 & 0.013518 & -0.575783 \\
\hline H4_3_2 & 0.281839 & 0.313491 & -0.391824 \\
\hline H4 _3_3 & -0.252204 & 0.038722 & -0.743068 \\
\hline H4_3_4 & 0.342394 & 0.246027 & -0.349393 \\
\hline C4_4 & 0.214413 & 0.114945 & -0.509708 \\
\hline H4_4_1 & 0.228418 & 0.190425 & -0.496222 \\
\hline H4_4_2 & 0.141492 & 0.062025 & -0.467591 \\
\hline H4_4_3 & 0.272312 & 0.106787 & -0.457289 \\
\hline H4_4_4 & 0.214746 & 0.101117 & -0.620706 \\
\hline
\end{tabular}

single crystal of orthoenstatite $\left[\left(\mathrm{Mg}_{1.93} \mathrm{Fe}_{0.06}\right)\left(\mathrm{Si}_{1.93}, \mathrm{Al}_{0.06}\right) \mathrm{O}_{6}\right.$, Pbca space group, $a=8.8117(2), b=5.18320(10)$, and $c=18.2391(3) \AA]$. The same calibration crystal was used at both beamlines. The structure was solved with the SHELXT structure solution program [25] using intrinsic phasing and refined with the JANA 2006 program [26]. Raman spectra were collected using the GSECARS Raman System [27] with the excitation wavelength of $532 \mathrm{~nm}$ and at the Earth and Planets Laboratory of Carnegie (EPL) using a 488-nm excitation line (e.g., Ref. [28]). Pressure was determined based on the shift of the $R 1$ ruby fluorescence line [29].

Ab Initio Random Structures Searching (AIRSS) [30] was performed using the CASTEP code [31] to explore the structures of the HP methane with the rhombohedral symmetry (the unit cells with up to 87 atoms were explored), with a subsequent relaxation using density functional theory (DFT) calculations [31]. Raman spectra were computed using the density functional perturbation theory (DFPT) + finite differences method, using the default CASTEP 18 norm-conserving pseudopotentials with a $875-\mathrm{eV}$ energy cutoff, and a $k$-point grid denser than $0.05 \times 2 \pi / \AA$.

\section{RESULTS AND DISCUSSION}

At pressures below $\sim 16 \mathrm{GPa}$, the diffraction pattern of $\mathrm{CH}_{4}$ (Fig. 1) can be indexed with a body-centered-cubic unit cell of phase $B$ (Table S1 and Fig. S3 [23]). Structure solution and refinement is in agreement with the model reported by Maynard-Casely et al. [15]. Due to weak scattering of hydrogen atoms, we were able to refine the positions of carbon atoms only. The unit cell contains four independent atomic sites: $\mathrm{C} 1, \mathrm{C} 2, \mathrm{C} 3$, and $\mathrm{C} 4$ occupying the positions $2 a, 24 g, 8 c$, and $24 \mathrm{~g}$, respectively. Therefore, there are $58 \mathrm{CH}_{4}$ molecules in the unit cell.

Further compression leads to a noticeable lattice distortion at above $18 \mathrm{GPa}$ and the unit cell can no longer be considered cubic. This distortion is accompanied by the appearance of new modes in the Raman spectrum (Fig. 2 and Fig. S2 of the Supplemental Material [23]) in agreement with the previous observations $[16,17]$ at similar pressure conditions. The most plausible indexing of the diffraction patterns of a methane high-pressure phase (HP) above $18 \mathrm{GPa}$ can be performed using a rhombohedral unit cell (Tables I and II). Symmetry-lowering transitions from cubic $I-43 m$ to rhombohedral symmetry may follow $\Gamma_{4}$ or $\Gamma_{5}$ irreducible representations with the following transformation of the lattice parameters:

$$
\left(\begin{array}{l}
\mathbf{a}_{\boldsymbol{R}} \\
\boldsymbol{b}_{\boldsymbol{R}} \\
\boldsymbol{c}_{\boldsymbol{R}}
\end{array}\right)=\left(\begin{array}{ccc}
-1 & 1 & 0 \\
0 & -1 & 1 \\
1 / 2 & 1 / 2 & 1 / 2
\end{array}\right)\left(\begin{array}{l}
\boldsymbol{a}_{\boldsymbol{c}} \\
\boldsymbol{b}_{\boldsymbol{c}} \\
\boldsymbol{c}_{\boldsymbol{c}}
\end{array}\right)
$$

where $\boldsymbol{a}_{\mathrm{R}} \boldsymbol{a}_{\mathrm{R}}, \boldsymbol{b}_{\mathrm{R}}, \boldsymbol{c}_{\mathrm{R}}$ are lattice vectors of the rhombohedral unit cell (hexagonal setting), while $\boldsymbol{a}_{\mathrm{c}}, \boldsymbol{b}_{\mathrm{c}}, \boldsymbol{c}_{\mathrm{c}}$ are lattice vectors of the cubic unit cell. The $\Gamma_{4}$ distortion leads to the space group symmetry $R 3 m$ (no. 160), while $\Gamma_{5}$ leads to $R 3$ (no. 146). We should note that at pressures above $30 \mathrm{GPa}$ we see a slight departure of the lattice parameters from $R$-centered hexagonal lattice $\left(a=b, \alpha=\beta=90, \gamma=120^{\circ}\right)$ suggesting a further monoclinic distortion. However, the degree of this distortion is not reproducible between two experiments (Fig. S4). Therefore, we conclude that this effect is related to the development of nonhydrostatic stresses in the sample, which is unavoidable at very high pressures and especially for very 

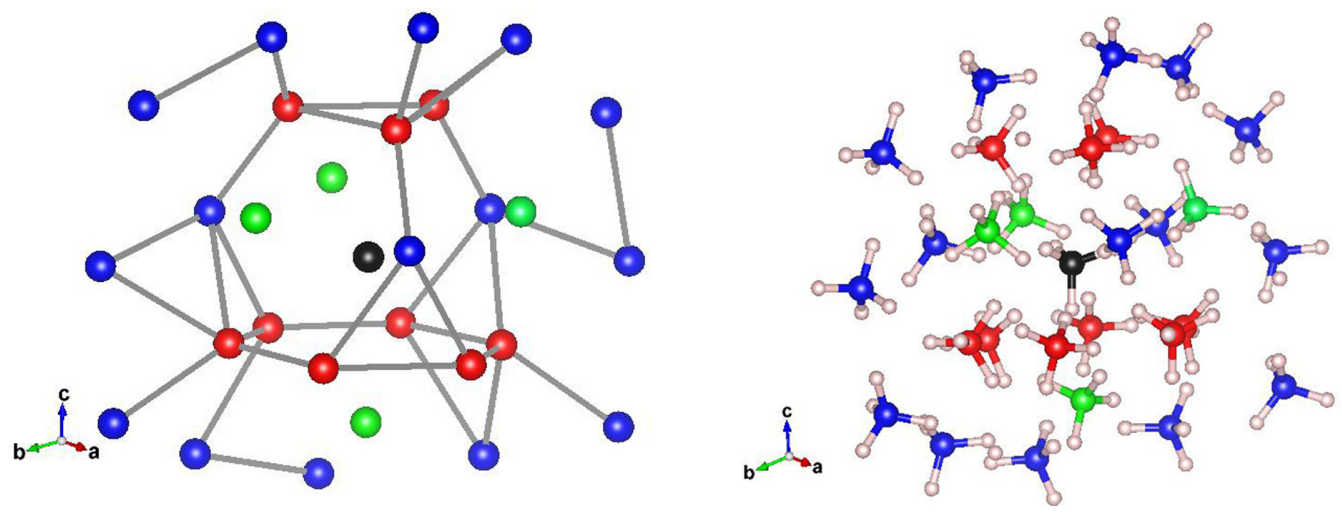

FIG. 4. Crystal structure of $\mathrm{HP}-\mathrm{CH}_{4}$ at $35 \mathrm{GPa}$. Black, red, green, and blue balls represent the positions of $\mathrm{C} 1, \mathrm{C} 2, \mathrm{C} 3$, and $\mathrm{C} 4 \mathrm{carbon}$ atoms, respectively; small pinkish circles are hydrogen atoms. Left panel shows $\mathrm{C}$ atoms only; gray lines connect the nearest $\mathrm{C}$ atoms with a cutoff of $3.08 \AA$.

soft crystals like methane. We therefore treat the high-pressure phase of methane as a single $R 3$ phase.

We have tested both $R 3$ and $R 3 m$ models (Table I). Although the $R 3$ model has smaller agreement factors, they are not significantly different from those of the $R 3 m$ model. We have performed a Hamilton significance test in order to check if the improvement of the agreement factors for the $R 3$ model may be considered significant [32]. For this test, both models were refined using the same set of reflections averaged based on the $R 3$ symmetry. Based on the Hamilton test, the $R 3$ model is preferable with the $75-90 \%$ confidence level depending on the pressure point. We, therefore, cannot completely reject the $R 3 m$ model based on the XRD data, but can conclude that the $R 3$ model is more likely (Table I).

In order to complete the structure solution and determine the positions of hydrogen atoms, theoretical structure optimizations have been performed at various pressures (Fig. 3). First, we performed an $a b$ initio random structure search (AIRSS) using the CASTEP code [31]. All calculations used the Perdew-Burke-Ernzerhof functional [33] together with the DFT- $D$ dispersion correction scheme of Tkatchenko and Scheffler [34]. Among a plethora of phases marginally different in enthalpies, two structures emerged having $R 3$ symmetries and 29 molecules in the primitive unit cell [R3-I and $R 3$-II in Fig. 2(a)] in agreement with the experimental HP phase (Fig. 4). Both calculated $R 3$ structures have equivalent carbon substructures and slightly different orientations of $\mathrm{CH}_{4}$ molecules centered at the $\mathrm{C} 4$ atoms. According to the theoretical calculations both $R 3$ structures are not the most energetically favorable at $0 \mathrm{~K}$. However, if temperature effects are taken into account, the calculated Gibbs free energies show that the R3-II phase becomes the most favorable above $260 \mathrm{~K}$ at $25 \mathrm{GPa}$, which perfectly agrees with the experiment [Fig. 3(b)]. A monoclinic distortion noticed experimentally is found to be energetically unfavorable. Below we will mainly discuss the most energetically favorable $R 3$ phase ( $R 3$-II, Table III).

The unit cell of HP-CH 4 (Fig. 4 and Fig. S5 of the Supplemental Material [23]) contains 11 symmetry independent carbon atoms with atoms $\mathrm{C} 1 \_1$ and $\mathrm{C} 3$ _1 occupying positions $3 a$, with the rest occupying positions $9 b$. Here, the $\mathrm{C}$ atoms are named following the numbering for phase $B$ [15] to show the relation between $B$ and HP phases, i.e., the atom $\mathrm{C} 3 \_1$ is generated from the atom $\mathrm{C} 3$ of the $B$ phase.

Figure 4 shows the main motif of the most energetically favorable $R 3$ structure. The C1_1 and C3_1 atoms that lie on the threefold axis possess a distorted tetrahedral icosioctahedral environment. This polyhedron can be understood in the following way (see bottom of the Fig. S5 [23]): the atom C3_1 is coordinated by two six-membered rings, one of which is planar and capped, while the other is in a chair arrangement capped by a triangle. The packing of $\mathrm{HP}-\mathrm{CH}_{4}$ is primarily defined by intermolecular $\mathrm{H}-\mathrm{H}$ interactions. The histogram of H-H distances has two distinct peaks (Fig. S6 [23]). The sharp peak at $\sim 1.75 \AA$ corresponds to intramolecular $\mathrm{H}-\mathrm{H}$ distances and almost does not shift with pressure as the molecules remain almost rigid under compression. The broad maxima at $\sim 2.05$ and $\sim 1.95 \AA$ at 20 and $30 \mathrm{GPa}$, respectively, correspond to closest intermolecular $\mathrm{H}$...H contacts. These distances are in a good agreement with shortest intramolecular $\mathrm{H}$.... contacts in compressed hydrogen [35].

The $v_{1}$ and $v_{3} \mathrm{C}-\mathrm{H}$ stretching Raman modes become composite (see also Refs. [16,17]) in phases $B$ and HP suggesting either site symmetry or vibrational splitting. The Raman spectra collected at $25 \mathrm{GPa}$ in the HP phase show that each of the $v_{1}$ and $v_{3}$ multifold can be well represented by four peaks (Figs. 2 and 5) except at $36 \mathrm{GPa}$, where an additional weak fifth peak tentatively assigned to the $v_{3}$ fundamental is seen. On the other hand, theoretical calculations of the Raman activity of the HP phase performed in this work show a larger number of components, which correspond to various molecular sites as the analysis for the $v_{1}$ modes shows (Fig. 5). However, the splitting pattern and value are qualitatively similar to the experimental; moreover, some theoretically calculated peaks are very close to each other making them difficult to resolve in the experiment; this likely results in broadened peaks, which consist of many components. A prominent feature of the $v_{1}$ Raman spectra is a well split off low frequency peak that theory predicts is due internal vibrations of molecules involving $\mathrm{C} 1$ and $\mathrm{C} 3$ carbon in $3 a$ sites, which are characterized by relatively more uniform in length and overall longer intermolecular distances (Fig. S7 [23]). This distinction results in a well-separated low-frequency $v_{1}$ component, which can be also seen in 

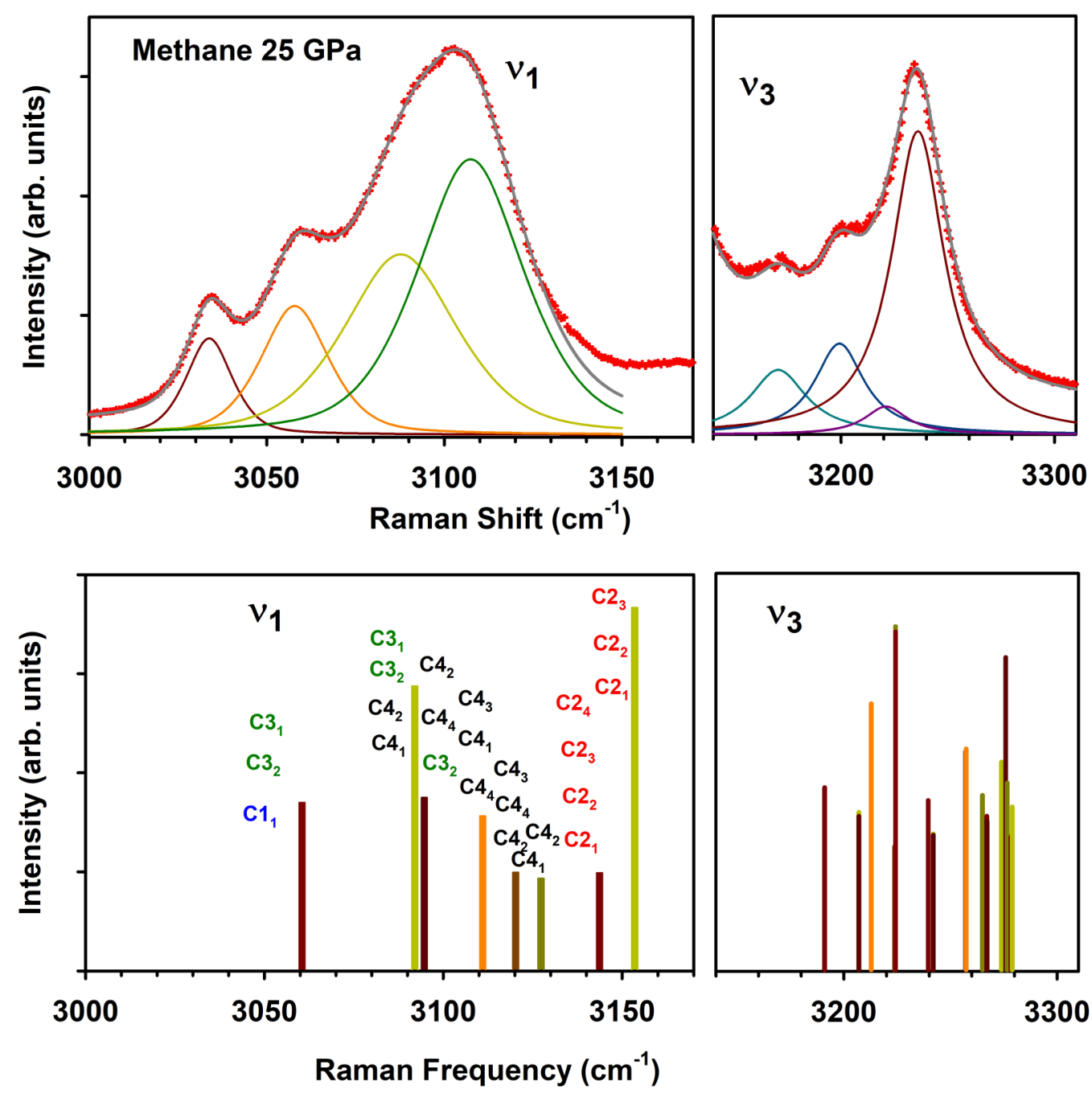

FIG. 5. Raman spectra of $v_{1}$ (left panels) and $v_{3}$ (right panels) stretching modes. The top panels are the spectrum measured at 25 GPa (488 nm excitation). Crosses are the data and gray line is the best fit to the Voigt oscillator model; variable color lines show the individual $v_{1}$ and $v_{3}$ components. The bottom panel shows the theoretically computed spectra at the same pressure. The labels (carbon atom sites) show the assignment of the peaks to the molecules of different kind. The experimental and theoretical peaks in the top and the bottom panels only slightly mismatch demonstrating a good correspondence of experiment and theory.

phase $B$ with similar carbon site geometry save the lattice distortion.

The unit-cell parameters and symmetry of phases $B$ and HP obtained here experimentally and theoretically provide reliable information on the $P-V$ equation of state of methane (Fig. 6). Our results agree well with the previous determinations based on single-crystal $[13,15]$ and powder $[19,20,36]$ XRD. The cubic unit cell of phase $B$ shows a distortion in the HP phase, which first appears small but then increases with pressure (Fig. S4 [23]). Please note that pre- $B$ phase, which appears on fast compression of phase $A$, was indexed as a simple cubic lattice $[19,20]$; this phase is not examined with XRD here (but was detected by Raman spectroscopy to coexist with HP phase in the experiment no. 2 up to the highest pressure investigated) but it is clearly different from the HP phase of this study. HP and pre- $B$ methane phases have very close volumes per the formula unit (Fig. 6) if one assumes that pre- $B$ has 21 molecules in the unit cell as in phase $A$. Unlike previously reported modifications of the HP phase based on changes in a number of $v_{1}$ and $v_{3}$ Raman components [16,17], our direct structural data (Fig. 1, Tables I and II) and analysis indicate that there exists only a single HP phase up to $71 \mathrm{GPa}$ (cf. Refs. [16,17]), where the rhombohedral distortion gradually increases with pressure (Fig. S4 [23]). Consistently, we also find (by fitting the spectra using an oscillator models, Figs. 2 and 5) that the number of C-H Raman components remains essentially the same (cf. Refs. [16,17]) and their frequencies (Fig. 7) agree well with the results of previous Raman investigations [16,17]. The pressure dependencies of all the Raman frequencies can be approximated by sublinear curves (Fig. 7). Previous Raman investigations $[16,17]$ reported minor changes in the spectra (peak splitting and changes in peak intensities) and in the $P$ dependence of the $\mathrm{C}-\mathrm{H}$ stretch frequencies. However, these changes do not signify the phase transitions, and, moreover, the inferred phase boundaries were inconsistent between these two investigations. The Raman peaks may split because they consist of a number of components, which have different 

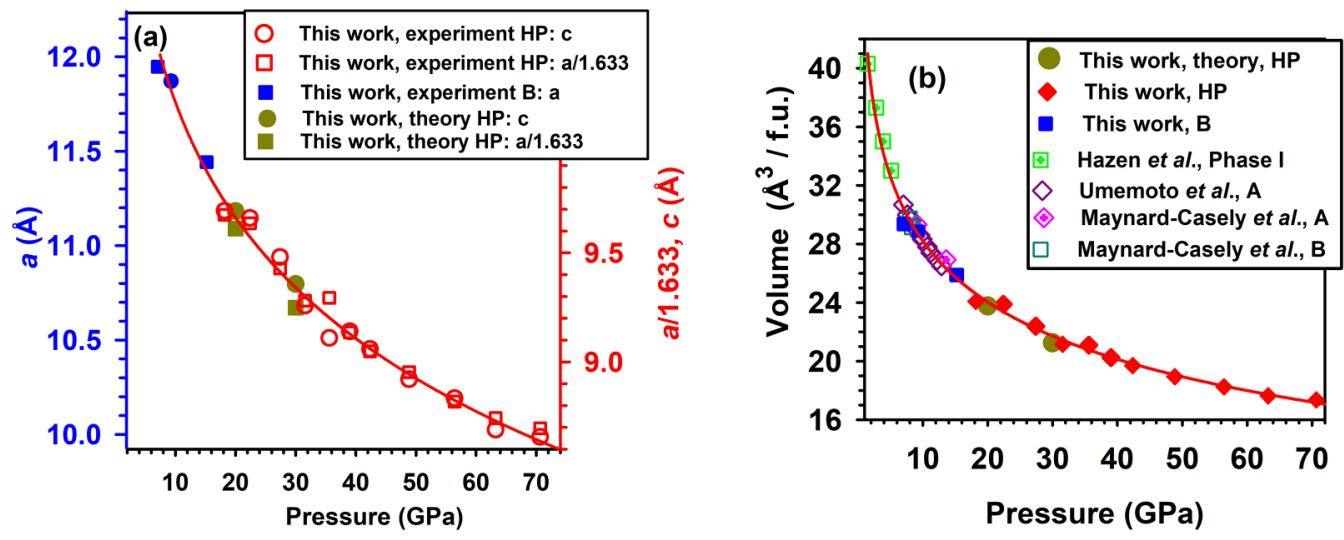

FIG. 6. Lattice parameters (a) and volumes per formula unit (b) of methane as a function of pressure. Note two vertical scales in the panel (a) for phases $B$ (blue) and HP (red) which are scaled by a factor of $\sqrt{3} / 2$ reflecting the difference in the unit cell dimensions. Also, the parameter $\boldsymbol{a}$ of the HP phase is divided by a factor of 1.633. Our experimental and theoretical volumes (b) are compared to previous experiments in phases I, $A, B$, pre-B, and HP [12,13,15,19,20]. The unit cell volume of pre-B methane of Ref. [20] was assumed to contain 21 formula units. The lines are guides to the eye.

pressure slopes so they become separated with pressure, while their relative intensity may change due to a number of reasons including the mode coupling and modification in the orientational order.

The unit cell volume changes smoothly through the $B$-HP transition suggesting a very small or no discontinuity. The theoretically computed lattice parameters and volumes are smaller than those determined theoretically, but the discrep-

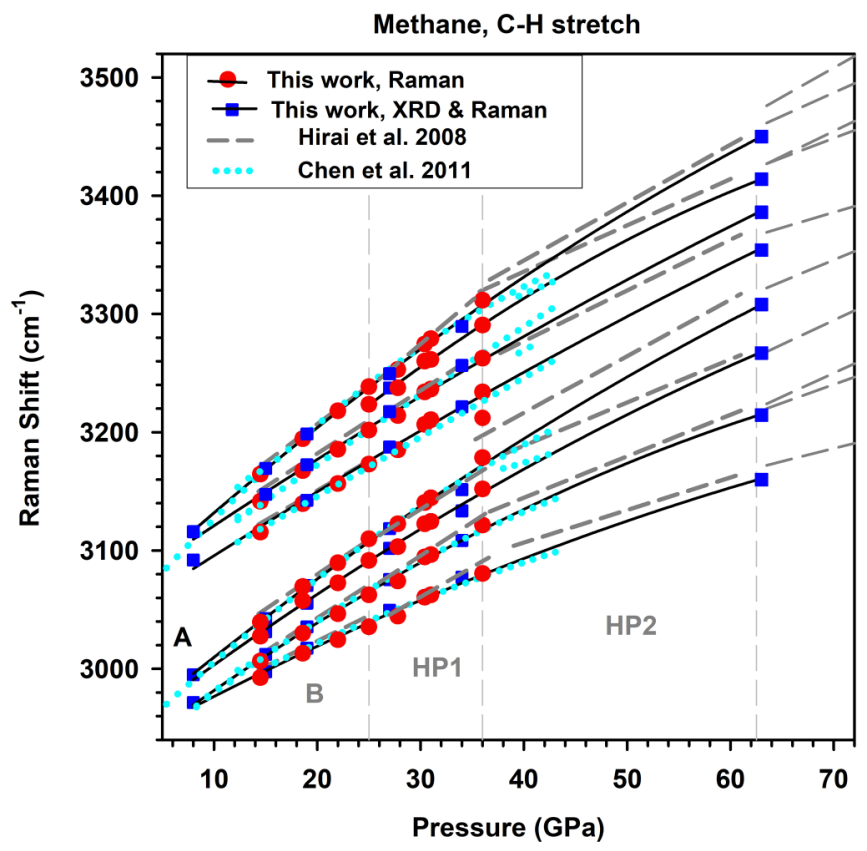

FIG. 7. Raman frequencies of the C-H stretching modes of methane as a function of pressure. Vertical dashed lines mark the phase transitions following Ref. [16]. The symbols are the results of two experiments of this work (solid line is the guide to the eye), while dashed and dotted lines represent the results of previously published works [16,17]. The data between 36 and 63 GPa could not be taken because the experiment to $63 \mathrm{GPa}$ was performed at the $\mathrm{x}$-ray synchrotron beamline, so the Raman spectra could be only measured at the last pressure point. ancy is within $3 \%$, which commonly is considered acceptable. However, this small difference may indicate that HP methane may still be partially disordered, which also corroborates with smaller (by some 20\%) observed values of $v_{1}$ and $v_{3}$ multifolds splitting compared to the computed ones (Fig. 5). It is also worth noting that the measured and calculated Raman frequencies agree quite well, within $1.5 \%$, demonstrating the validity of approximations made for computing the Raman spectra.

\section{CONCLUSIONS}

Our concerted experimental and theoretical investigation determined the structure of the high-pressure HP phase of methane including the positions of hydrogen atoms. The structure is rhombohedral (space group $R 3$ ) with 87 molecules in the unit cell. This extraordinary complex structure of approximately spherical molecules is in a drastic contrast with simple fcc (hcp) structures of noble gas solids. Moreover, the theoretically predicted orientationally ordered structures with smaller unit cell and with well elaborated intermolecular coupling schemes also do not capture the real structure. This indicates that the observed structure represents a balance between enthalpy and entropy terms thus suggesting that some molecular sites remain orientationally disordered; this makes this structure analogous to clathrates, where host and guest molecules are the same.

Our direct single-crystal XRD results suggest that there is only one high-pressure phase (HP) of methane between 20 and $71 \mathrm{GPa}$ in contrast to previous works, which suggested up to three HP phases based on the results of Raman spectroscopy. The HP phase investigated here can coexist with a cubic pre- $B$ phase investigated previously, the detailed structure of which still remains unknown.

\section{ACKNOWLEDGMENTS}

Parts of this research were carried out at the Extreme Conditions Beamline (P02.2) at DESY, a member of Helmholtz Association (HGF). Portions of this work were performed 
at HPCAT (sector 16) of the Advanced Photon Source (APS), Argonne National Laboratory. HPCAT operations are supported by DOE-NNSA's Office of Experimental Sciences. Concomitant Raman spectroscopy experiments were performed at GeoSoilEnviroCARS (The University of Chicago, Sector 13), Advanced Photon Source (APS), Argonne National Laboratory. GeoSoilEnviroCARS is supported by the National Science Foundation - Earth Sciences (EAR - 1634415) and Department of Energy GeoSciences (Grant No. DE-FG02-94ER14466). The Advanced Photon Source is U.S. Department of Energy (DOE) Office of Science
User Facility operated for the DOE Office of Science by Argonne National Laboratory under Contract No. DE-AC0206CH11357. C.J.P. acknowledges financial support from the Engineering and Physical Sciences Research Council (Grant No. EP/P022596/1). M.M.-C. is grateful for computational support from the UK national high performance computing service, ARCHER, for which access was obtained via the UKCP consortium and funded by EPSRC Grant No. EP/P022561/1. M.B.'s research was sponsored by the Army Research Office and was accomplished under the Cooperative Agreement No. W911NF-19-2-0172.
[1] P. Hebert, A. Polian, P. Loubeyre, and R. Le Toullec, Phys. Rev. B 36, 9196 (1987).

[2] N. W. Ashcroft, Phys. Rev. Lett. 92, 187002 (2004).

[3] E. Snider, N. Dasenbrock-Gammon, R. McBride, M. Debessai, H. Vindana, K. Vencatasamy, K. V. Lawler, A. Salamat, and R. P. Dias, Nature (London) 586, 373 (2020).

[4] W. B. Hubbard, Planetary Interiors (Van Nostrand Reinhold, New York, 1984).

[5] C. Cavazzoni, G. L. Chiarotti, S. Scandolo, E. Tosatti, M. Bernasconi, and M. Parrinello, Science 283, 44 (1999).

[6] S. Ninet, F. Datchi, and A. M. Saitta, Phys. Rev. Lett. 108, 165702 (2012).

[7] V. B. Prakapenka, N. Holtgrewe, S. S. Lobanov, and A. F. Goncharov, Nat. Phys. (2021), doi: 10.1038/s41567-02101351-8.

[8] S. S. Lobanov, P.-N. Chen, X.-J. Chen, C.-S. Zha, K. D. Litasov, H.-K. Mao, and A. F. Goncharov, Nat. Commun. 4, 2446 (2013).

[9] F. Ancilotto, G. L. Chiarotti, S. Scandolo, and E. Tosatti, Science 275, 1288 (1997).

[10] D. Kraus, J. Vorberger, A. Pak, N. J. Hartley, L. B. Fletcher, S. Frydrych, E. Galtier, E. J. Gamboa, D. O. Gericke, S. H. Glenzer, E. Granados, M. J. MacDonald, A. J. MacKinnon, E. E. McBride, I. Nam, P. Neumayer, M. Roth, A. M. Saunders, A. K. Schuster, P. Sun, T. van Driel, T. Döppner, and R. W. Falcone, Nat. Astron. 1, 606 (2017).

[11] G. Gao, A. R. Oganov, Y. Ma, H. Wang, P. Li, Y. Li, T. Iitaka, and G. Zou, J. Chem. Phys. 133, 144508 (2010).

[12] R. M. Hazen, H. K. Mao, L. W. Finger, and P. M. Bell, Appl. Phys. Lett. 37, 288 (1980).

[13] H. E. Maynard-Casely, C. L. Bull, M. Guthrie, I. Loa, M. I. McMahon, E. Gregoryanz, R. J. Nelmes, and J. S. Loveday, J. Chem. Phys. 133, 064504 (2010).

[14] R. Bini, L. Ulivi, H. J. Jodl, and P. R. Salvi, J. Chem. Phys. 103, 1353 (1995).

[15] H. E. Maynard-Casely, L. F. Lundegaard, I. Loa, M. I. McMahon, E. Gregoryanz, R. J. Nelmes, and J. S. Loveday, J. Chem. Phys. 141, 234313 (2014).

[16] H. Hirai, K. Konagai, T. Kawamura, Y. Yamamoto, and T. Yagi, Chem. Phys. Lett. 454, 212 (2008).

[17] P.-N. Chen, C.-S. Zha, X.-J. Chen, J. Shu, R. J. Hemley, and H.-k. Mao, Phys. Rev. B 84, 104110 (2011).
[18] R. Bini and G. Pratesi, Phys. Rev. B 55, 14800 (1997).

[19] S. Umemoto, T. Yoshii, Y. Akahama, and H. Kawamura, J. Phys.: Condens. Matter 14, 10675 (2002).

[20] L. Sun, W. Yi, L. Wang, J. Shu, S. Sinogeikin, Y. Meng, G. Shen, L. Bai, Y. Li, J. Liu, H. k. Mao, and W. L. Mao, Chem. Phys. Lett. 473, 72 (2009).

[21] L. Sun, Z. Zhao, A. L. Ruoff, C.-S. Zha, and G. Stupian, J. Phys.: Condens. Matter 19, 425206 (2007).

[22] I. Kantor, V. Prakapenka, A. Kantor, P. Dera, A. Kurnosov, S. Sinogeikin, N. Dubrovinskaia, and L. Dubrovinsky, Rev. Sci. Instrum. 83, 125102 (2012).

[23] See Supplemental Material at http://link.aps.org/supplemental/ 10.1103/PhysRevB.104.184105 for methods, supplemental figures (S1-S5), and tables (S1-S5), and references [27-30,32,34].

[24] Rigaku Oxford Diffraction, CrysAlisPro Software System, Version 1.171 (Rigaku Corporation, Oxford, UK, 2021).

[25] G. M. Sheldrick, Acta Crystallogr., Sect. A: Found. Adv. 71, 3 (2015).

[26] V. Petricek, M. Dusek, and L. Palatinus, Z. Kristallogr. 229, 345 (2014).

[27] N. Holtgrewe, E. Greenberg, C. Prescher, V. B. Prakapenka, and A. F. Goncharov, High Pressure Res. 39, 457 (2019).

[28] A. F. Goncharov, P. Beck, V. V. Struzhkin, R. J. Hemley, and J. C. Crowhurst, J. Phys. Chem. Solids 69, 2217 (2008).

[29] H. K. Mao, J. Xu, and P. M. Bell, J. Geophys. Res.: Solid Earth 91, 4673 (1986).

[30] C. J. Pickard and R. J. Needs, J. Phys.: Condens. Matter 23, 053201 (2011).

[31] S. J. Clark, M. D. Segall, C. J. Pickard, P. J. Hasnip, M. I. J. Probert, K. Refson, and M. C. Payne, Z. Kristallogr. - Cryst. Mater. 220, 567 (2005).

[32] W. C. Hamilton, Acta Crystallogr. 18, 502 (1965).

[33] J. P. Perdew, K. Burke, and M. Ernzerhof, Phys. Rev. Lett. 77, 3865 (1996).

[34] A. Tkatchenko and M. Scheffler, Phys. Rev. Lett. 102, 073005 (2009).

[35] V. Labet, P. Gonzalez-Morelos, R. Hoffmann, and N. W. Ashcroft, J. Chem. Phys. 136, 074501 (2012).

[36] I. Nakahata, N. Matsui, Y. Akahama, and H. Kawamura, Chem. Phys. Lett. 302, 359 (1999). 